\title{
TENDENCIAS DE LA FECUNDIDAD EN ARAGON
}

\author{
Maria Carmen FAUS PUJOL \\ Universidad de Zaragoza
}

\begin{abstract}
Resumen.- La población aragonesa tiene su futuro demográfico muy comprometido . Así lo muestran los principales indicadores de fecundidad aplicados para su estudio. La explicación de la actual tendencia negativa debe buscarse en factores históricos y, sobretodo hoy, en factores sociales y comportamentales relativos al matrimonio y la procreacion.
\end{abstract}

Abstract: The demographic future of Aragon is very involved as explain the main fertility indicators calculated for this region. The present negative trend is owing to historical factors and, overcoat nowadays, behaviour factors related vith nuptiality and procreation.

Palabras clave: Natalidad.- Fecundidad.- Transición demográfica.

Sumario: Natalidad cada vez más baja.- Fecundidad extraordinariamente baja.- Factores explicativos de la caida de la fecundidad en Aragón.- Algunas conclusiones.- Bibliografía.

El estudio de la fecundidad, como elemento esencial del crecimiento natural de la población, guarda estrecha relación con la estructura demográfica y en particular con el reparto por edad de la población femenina, y con la actitud y el comportamiento de la población frente a la natalidad, que en España, y más concretamente en Aragón, son actualmente restrictivos.

Antes de entrar en el análisis de las tendencias de la fecundidad en Aragón, conviene recordar algunos datos. Aragón ocupa el 9,44\% del territorio español; su población equivale al 3,1\% de la española; la densidad es de solo 25 habitantes por kilómetro cuadrado, lo cual coloca a nuestra región en la España semidespoblada; y con un indice de crecimiento intercensal reducido, $0,36 \%$ anual, es una región de escaso peso demografico a escala nacional.

Algo más de la mitad de su población se concentra en la capital regional. La desigual distribución de la población repercute en la estructura demográfica, y por tanto en las diferencias de fecundidad que se observan entre las provincias y las capitales, y entre unas 
comarcas y otras. Además la emigracion ha jugado un papel de primer orden en la configuración de la estructura demográfica por sexo y edad.

En los últimos 10 años los grandes flujos migratorios que caracterizaron a esta región hasta 1970 se han detenido. Sin embargo la movilidad interna continúa. Se produce una migración por "goteo" de caracter interprovincial, y desde las áreas rurales a las urbanas. Todo esto explica el envejecimiento y la reducción de la población femenina en las áreas rurales y en consecuencia la disminución de la fecundidad de las mismas.

\section{NATALIDAD CADA VEZ MAS BAJA}

La disminución de la natalidad forma parte de la transición demográfica de los países desarrollados de Europa occidental. Aunque con cierto retraso respecto de Europa, el descenso de natalidad no es un fenómeno nuevo en nuestro paŕs. Ya desde la segunda mitad del siglo XIX se aprecia una tendencia a la baja en la natalidad española, tímidamente al principio (pasando del $38 \%$ en 1850 al $35 \%$ en 1900) y de modo mas evidente en lo que va de siglo (21\%o en 1950, $19 \%$ en 1975 y $11 \%$ en 1985). El ritmo del descenso es particularmente acelerado desde 1977 con una disminución algo superior al $1 \%$ anual.

TASAS DE NATALIDAD $(, 000)$

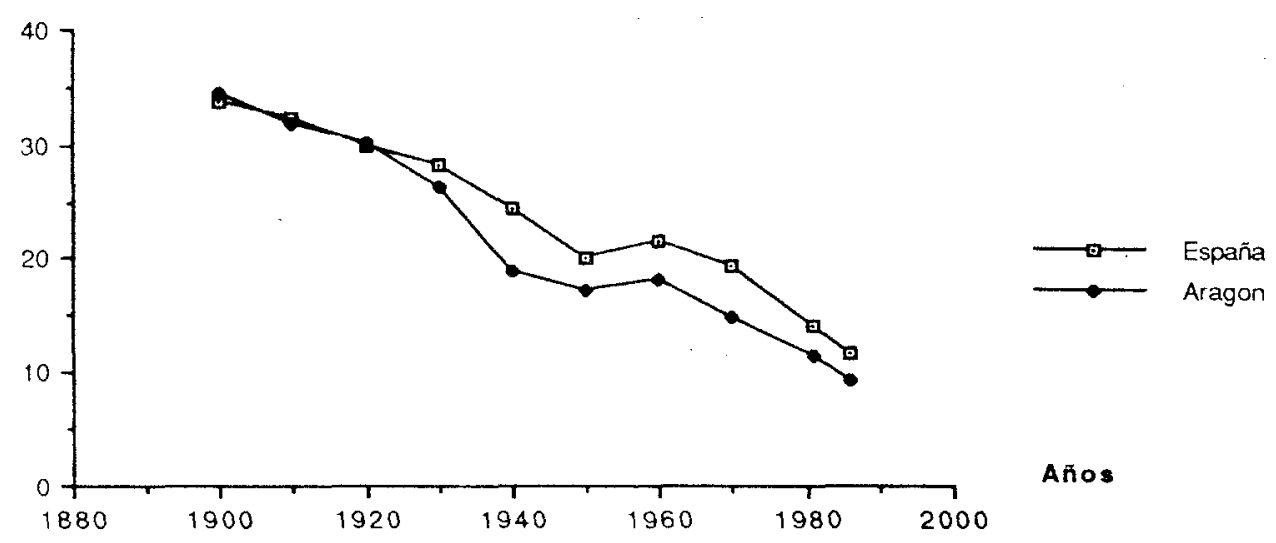


En el contexto regional de Aragón, la natalidad ha seguido la misma tendencia aunque con ciertas particularidades:

a) Aragón se cuenta entre las primeras regiones españolas en las que se pone de manifiesto la disminución de la natalidad, no solamente como consecuencia del desarrollo de la transición demográfica, sino sobre todo por la intensidad de las migraciones anteriormente mencionadas. A partir de 1915 la natalidad aragonesa se aleja cada vez mas de la española hasta alcanzar una diferencia máxima de casi cinco puntos en 1970-75.

b) En 1975 -fecha tomada como punto de referencia para seguir la evolución reciente de la fecundidad regional- la natalidad aragonesa se mantenía bastante por debajo de la española como se acaba de decir. Sus valores medios, alrededor del $14 \%$ equivalen a la media española de 1981. En 1981 se mantiene la tendencia a la baja, con una natalidad del $11,48 \%$ frente al $14,13 \%$ de la española. Lo mismo sucede en 1986 aunque la diferencia con la media española se hace cada vez menor $(11,72 \%$ en España, $9,36 \%$ en Aragón), ya que poco a poco todas las regiones españolas van acusando la disminución de la natalidad, aunque las causas no sean las mismas en todas partes.

c) Los datos globales enmascaran notables diferencias entre la natalidad considerada a nivel provincial y la referida a la capital de cada provincia. La natalidad de las capitales provinciales puede equipararse a la media del país; en cambio, la de las provincias quedaba a una distancia del orden de 7-8 puntos por debajo en 1975; y de 4 a 8 en 1981.

d) De 1975 a 1981 la natalidad disminuye tanto en las provincias como en las capitales, tendencia que sigue en la actualidad. Zaragoza es la provincia que acusa mayor descenso, especialmente en su capital, 8,91\% entre 1975 y 1986. Si tenemos en cuenta que en la ciudad de Zaragoza se concentra la mayor parte de la población aragonesa y que su grado de juventud es ligeramente superior al resto de la región, se comprende que las razones del descenso de natalidad no se relacionan únicamente con la estructura demográfica. 
Tasas de Natalidad (\%)

Provincias

$\underline{1975}$

$$
1981
$$

$\underline{1986}$

10,23

6,26

19,73

8,02

17,41

8,28

Capitales

$\underline{1981}$

1986

\begin{tabular}{lrrrrrr} 
Huesca & 12,23 & 10,23 & 6,26 & 19,73 & 14,60 & 20,08 \\
Teruel & 10,23 & 9,56 & 8,02 & 17,41 & 17,39 & 10,70 \\
Zaragoza & 11,08 & 8,91 & 8,28 & 19,36 & 13,39 & 10,45 \\
\hline
\end{tabular}

Fuente: IN.E.

e) Por lo que se refiere a las comarcas aragonesas,en 1975-81 la natalidad presenta diferencias de hasta 7 puntos entre las que tenían tasas mas reducidas (Tierra de Belchite 3,41\%o;Mora-Gúdar 5,51\%o) y las mas elevadas (Maestrazgo Turolense 11,07\%o y Parameras Montalbinas 13,06\%o). De 1981 a 1986 el descenso de la natalidad es todavía mas acusado, y la distancia entre las tasas mínimas y las máximas alcanza 9 puntos (Val de Ayerbe-Gállego Medio 2,37\%, Parameras Montalbinas 11,72\%o). Algunas excepciones a la tendencia general corresponden a las comarcas del centro de la región, situadas generalmente sobre el río Ebro que es el área regional más dinámica. Con todo, su variación positiva rara vez supera el $1 \%$.

Las consecuencias de esta situación son evidentes.La disminución de la natalidad ralentiza el crecimiento demográfico de la región y no hay renovación de la base de la pirámide de edades. Como la mortalidad es muy baja, aumenta el envejecimiento de la población con todo lo que lleva consigo. Así Aragón es una región de población envejecida, varios puntos más vieja que la población española.

Todo esto se ha repetido hasta la saciedad y no merece que nos detengamos en ello. Lo que nos interesa ahora es reflexionar sobre el descenso de natalidad aragonesa y las causas que han llevado a esta situación. Las pérdidas de población, fruto de la emigracion, han jugado su papel, pero no lo explican todo. El estudio de la fecundidad permite apuntar otras conclusiones. 


\section{FECUNDIDAD EXTRAORDINARIAMENTE BAJA}

La característica mas significativa de este hecho demográfico queda definida por los titulares de este apartado. La fecundidad en Aragón ha llegado a valores mínimos, cualquiera que sean los indicadores que se utilicen para analizarla.

La fecundidad, como la natalidad, ha sido moderadamente baja a lo largo de este siglo. Pero en la última década se ha producido una brusca caida tanto en las provincias, como en las ciudades y capitales provinciales. En general las actuales tasas globales de fecundidad están al nivel propio de los paises mas desarrollados y se acercan al $40 \%$. Pero en el caso de Aragón no puede decirse que la escasa fecundidad sea consecuencia del alto grado de desarrollo.

\section{Tasa global de fecundidad (\%o) (TGF)*}

\section{Provincias}

1975

\begin{tabular}{lrrrrrr} 
& 1975 & $\underline{1981}$ & $\underline{1986}$ & $\underline{1975}$ & $\underline{1981}$ & $\underline{1986}$ \\
Huesca & 69,12 & 56,94 & 34,54 & 89,19 & 69,58 & 68,95 \\
Zaragoza & 63,21 & 52,02 & 45,71 & 90,15 & 63,02 & 48,44 \\
Teruel & 61,17 & 59,85 & 47,25 & 84,23 & 85,44 & 39,39 \\
\hline
\end{tabular}

Fuente: IN.E.

\section{Capitales}

* Pone en relación el total de nacimientos con el de mujeres en edad fertil.

En el análisis de la fecundidad hemos seguido las recomendaciones de J. A. Coale (1969) y G. Tapinos (1985). Se han calculado los nacimientos teóricamente posibles mediante estandarización de las tasas de fecundidad por edad en la población de Aragón en 1981, tomada como población-tipo. Aplicadas esas tasas a cada una de las comarcas aragonesas en 1981 y 1986, se ha comprobado que en todos los casos el número de nacimientos reales queda bastante por debajo de los nacimientos teóricamente posibles, incluso aceptando los cambios socioeconómicos previstos por la transición demográfica. Excluyendo las tres capitales de provincia, en 1981 el número de nacimientos ocurridos 
fue un $14 \%$ menor que los esperados; en 1986 un $33 \%$ y en 1988 , según cifras provisionales, un $36 \%$.

TASA BRUTA DE NATALIDAD COMARCAL ( 1981 *

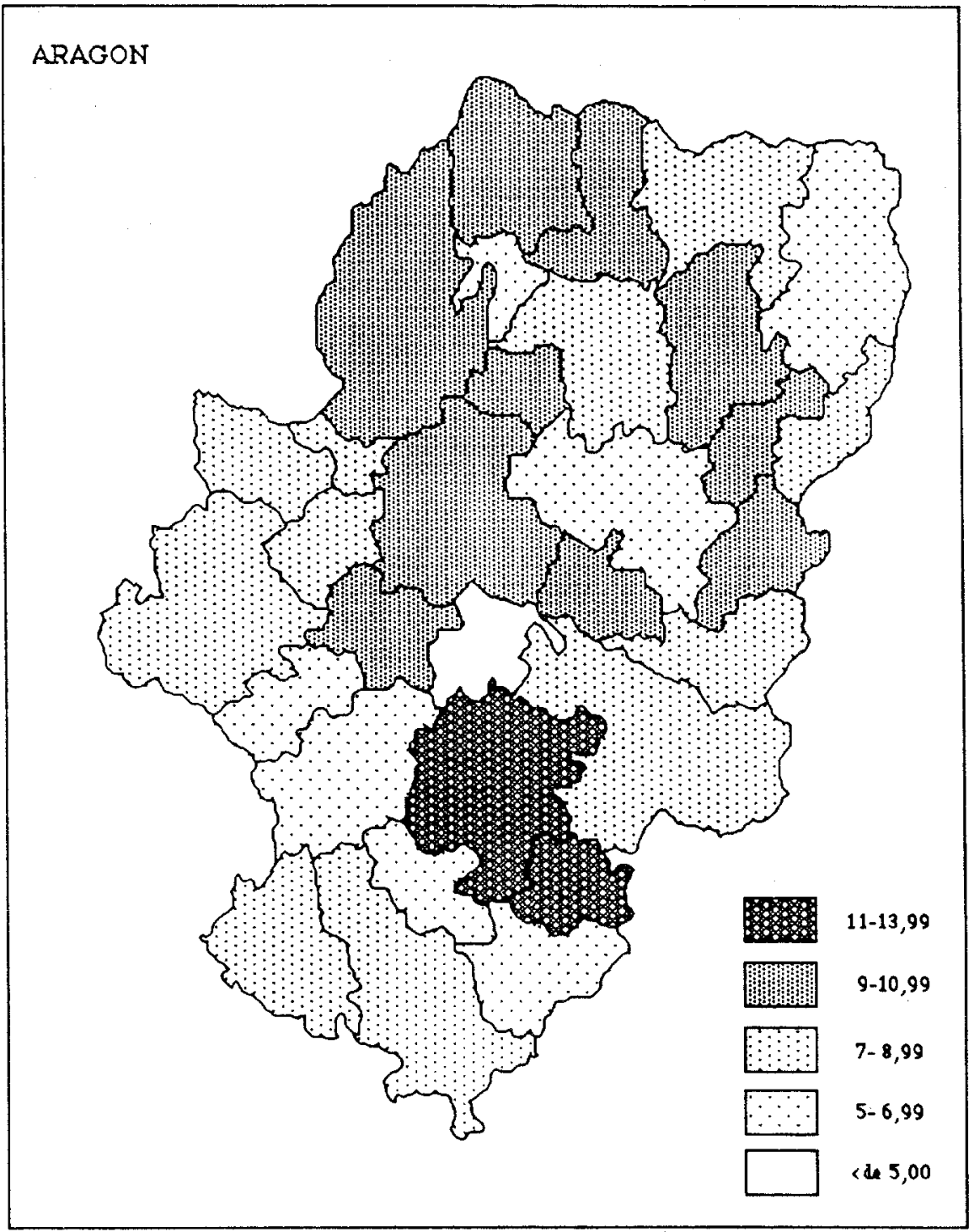

* División comarcal utilizada en la Geografia de Aragón, dirigida por A. Higueras. Ed. Guara. Zaragoza, 1982-1985. 


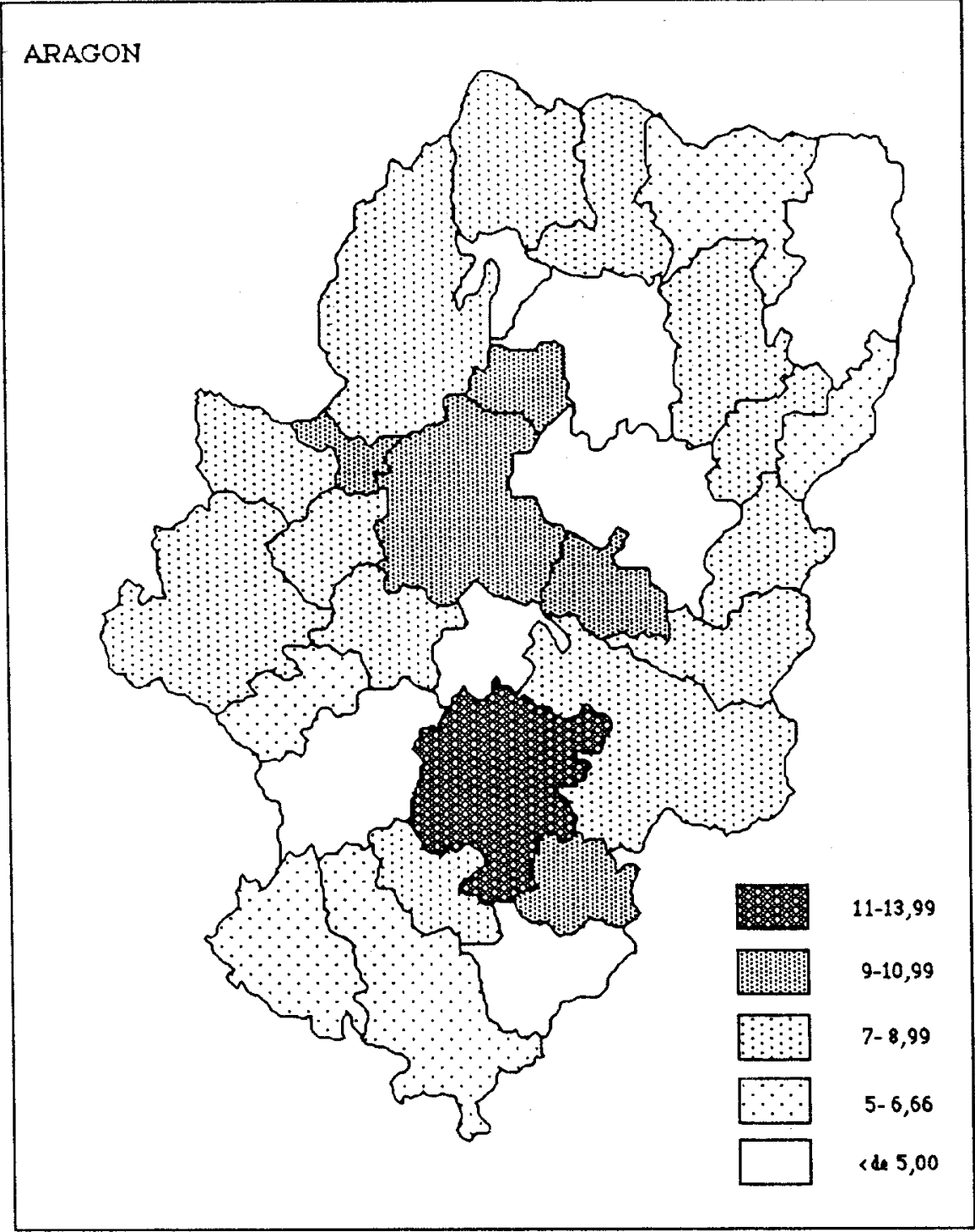


DIFERENCIA ENTRE LAS TASAS DE NATALIDAD DE 1981 Y 1986.(\%)

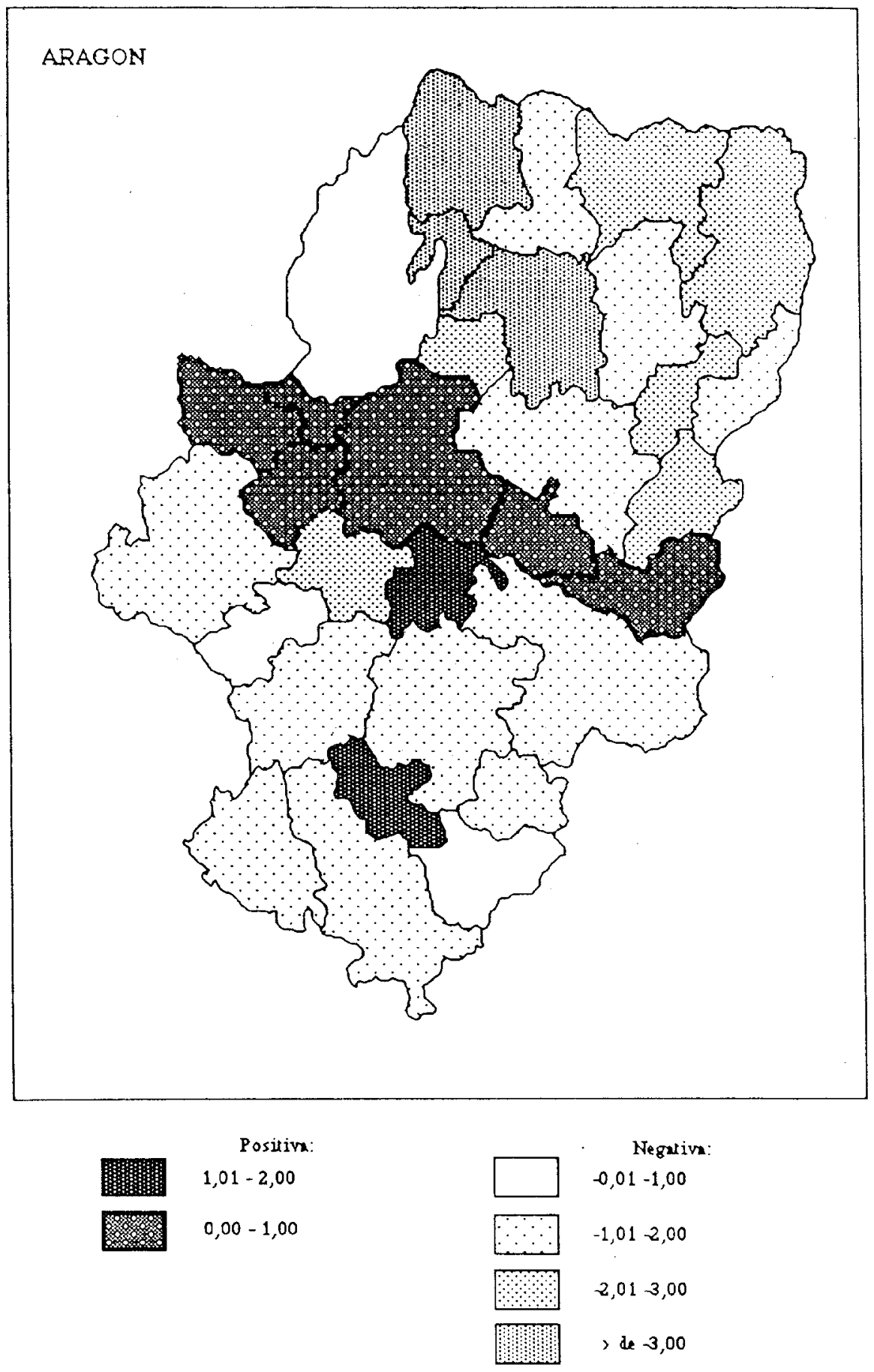


Estas cifras envuelven sin duda errores provenientes de lagunas estadísticas. Por eso es necesario hacer algunas precisiones para una adecuada valoración del análisis comparativo de las tasas globales de fecundidad calculadas en dos fechas distintas para las provincias y sus capitales.

Durante mucho tiempo, gran parte de los nacimientos acontecidos en la población residente en áreas rurales se censaban en las capitales. Ello se explica por la concentración en ellas de los centros hospitalarios de asistencia maternal. Aunque legalmente la inscripción de los neonatos debe hacerse en el lugar de residencia de la madre, no siempre sucede así. Nosostros hemos corregido los datos por encuesta directa, pero puede haber todavía algunos errores.

Por otra parte hay cierta resistencia a reconocer las pérdidas de población. En los últimos años se observa que muchos emigrantes, por razones muy diversas, se inscriben en el lugar de origen, aunque no sean residentes de facto. Esto suele estar bien visto por las autoridades municipales, pues las transferencias de dotaciones económicas se hacen de acuerdo con el número de habitantes de cada municipio. Este y otros inconvenientes hemos intentado resolverlos por encuesta directa, como se ha dicho, para evitar, en la medida de lo posible, las distorsiones que todo ello introduce en el cálculo de la fecundidad.

Mujeres en edad fecunda (15-44 años) en \% del total provincial

Provincias

1975

$\begin{array}{lrrrrrr} & 1975 & \underline{1981} & \underline{1986} & \underline{1975} & \underline{1981} & \underline{1986} \\ \text { Huesca } & 80,63 & 77,67 & 78,57 & 19,37 & 22,33 & 21,43 \\ \text { Zaragoza } & 29,06 & 26,55 & 25,83 & 0,94 & 73,45 & 74,17 \\ \text { Teruel } & 82,21 & 9,42 & 78,71 & 17,79 & 20,58 & 21,29\end{array}$

Capitales

Fuente: I.N.E. 
Eso explicaría la anomalía que se observa en las provincias de Huesca y Teruel donde las capitales dan las mayores tasas globales de fecundidad cuando la mayor parte de la población femenina en edad fecunda se reparte a lo largo de dichas provincias.

El comportamiento de Zaragoza capital es significativo en lo referente a la evolución de la actitud respecto de la natalidad. De 1975 a 1986 aumentó la concentración de la población femenina en edad fecunda, pero paradójicamente la TGF disminuye. Esto indica sin duda que se ha producido un cambio del comportamiento de los progenitores frente a la fecundidad.

La fecundidad de las comarcas se ve tambien afectada por el número de mujeres en edad fértil. La estructura por edad de la población femenina es muy desigual en unas comarcas y otras. En 1981 la tasa mínima de mujeres en edad fecunda era del 22,34 \%o y correspondia a una de las comarcas demográficamente mas deprimidas de la región (Tierra de Belchite); la máxima ascendía hasta $73,10 \%$, bastante por encima de la media regional, en las Parameras Montalbinas. En 1986 los polos opuestos eran la comarca de Val de Ayerbe-Gállego medio con 15,76\%o y de nuevo las Parameras Montalbinas con $63,00 \%$.

En los últimos años se ha hablado mucho de la incidencia de la natalidad extramatrimonial en la fecundidad, pero no ha sido posible entrar en detalle en el estudio de la fecundidad ilegítima a nivel de comarcas. En Aragón la mayor parte de la natalidad es legítima. En la actualidad han cambiado los criterios de legitimidad. Las Encuestas de Fecundidad realizadas por el Instituto Nacional de Estadística consideran que todos los nacimientos son legítimos, tanto si son matrimoniales como extramatrimoniales. En cualquier caso no creemos que ello influya en la fecundidad. Aunque se den casos de nacimientos desconocidos en el lugar de residencia de la madre, su número no debe ser tan elevado como para modificar las tasas de natalidad. En Aragón alrededor del $69 \%$ de las mujeres están casadas y en su mayoría mantienen una unión estable, pero su comportamiento respecto de la natalidad es restrictivo. Así lo prueba el número medio de hijos por familia.

Casi la mitad de las familias tienen de uno a dos hijos (uno el $18,85 \%$ y dos el $26,69 \%$ ), algo mas de un tercio no tienen ninguno $(35,08 \%)$ y son muy pocas las familias numerosas. La reducción del tamaño de la familia es consecuencia y causa de la escasa fecundidad, que tiende a decrecer todavía más, como consecuencia de un proceso 
de feedback negativo. De todo lo anterior se deduce, como decíamos antes, que en Aragón el deterioro de la fecundidad es un proceso que viene de atrás. Los indicadores coyunturales de fecundidad (o indicadores sintéticos de fecundidad) obtenidos por estandarización para anular el efecto de la estructura por edad, reflejan una situación regresiva ya en 1975, y muy regresiva a partir de 1981.

Así resulta que Aragón es una de las regiones españolas con los indicadores de fecundidad mas bajos de España. La media regional de 1975 estaba en el nivel de reemplazamiento considerado mínimo en los paises desarrollados: 2,2. En 1981 era solamente de 1,8 ; en 1986 apenas supera la unidad y en 1988 era de sólo el 0,88 . Si en 1981 la media española todavía llegaba al mínimo necesario para asegurar la renovación generacional, Aragón ya no alcanzaba ese mínimo.

En consecuencia y sin ánimo de ser pesimistas, en ninguna comarca aragonesa, dadas las actuales tasas de fecundidad está asegurado el reemplazo generacional. Las tasas brutas de reproducción que no tienen en cuenta la mortalidad indican lo mismo;la media es inferior a la unidad $(0,878)$. Finalmente nuestras previsiones al año 2000 (A. Higueras y M.C.Faus, 1988), son igualmente negativas ya que no se prevee un cambio de tendencia.

La reducción del número de nacimientos y de hijos por familia es evidente. Ahora hay que preguntarse si la disminución de la fecundidad obedece a una actitud contraria a la natalidad, que incidiría sobre el control de la fecundidad, si es consecuencia de la estructura demográfica, o de ambas cosas a la vez, que es nuestra tesis por el efecto de feedback negativo ya apuntado.

\section{FACTORES EXPLICATIVOS DE LA CAIDA DE LA FECUNDIDAD EN ARAGON}

El hecho de que la fecundidad en Aragón sea una de las mas bajas de España obliga a buscar factores explicativos de esta situación, por la trascendencia socioeconómica de la misma.

La teoría clásica de la transición demográfica, que relaciona los cambios de fecundidad con los niveles de desarrollo y la transformación de la función socioeconómica de la familia, no es del todo válida para la mayor parte de Aragón. En buena parte de la 
región el descenso de fecundidad se inició dentro de una economía predominantemente agraria cuyos cambios son mas recientes.

Cabe encuadrar la situación aragonesa dentro del proceso secular de la baja de la natalidad en España, pero eso no explica por qué ésta se inicia antes en Aragón, ni por qué su caida es mas fuerte que en la mayoría de las regiones españolas, exceptuando Cataluña. Tampoco se observan en Aragón ciclos de alta y baja fecundidad, que podrían explicar su singularidad. Ninguna de las teorías comunes aceptadas para explicar el descenso de la fecundidad son aplicables a nuestra región. La oferta de trabajo o la situación económica relativa, según las ideas de J.Grauman y R.Easterlin, tampoco influyen en la baja fecundidad aragonesa en este siglo, y sobre todo en los últimos diez años. Recientes estudios sobre la fecundidad y el paro en España (A.Garcia y M.J.Crespo, 1988), muestran que la tesis de Easterlin no se verifica en nuestro país. En Aragón coincide la tendencia a la baja de la fecundidad con bajas tasas de paro. Incluso este último desciende entre 1981 y 1986 en términos relativos respecto del conjunto de España (en 1981 el 2,7 \% del paro español, en 1986 el 2,5\% y en 1988 el 2,4\%).

Mayor influencia han tenido en algunas comarcas factores de tipo institucional por sus consecuencias en la movilidad de la población y en la estructura demográfica. Ese es el caso, por ejemplo, de la institución denominada del "heredero único" que dejaba el patrimonio familiar al primogénito o al primer hijo varón. Los demás hijos de la familia y sobre todo las mujeres, se veían obligados a ocuparse en otros sectores laborales y frecuentemente a emigrar.

La emigración, por afectar a la población joven, y en muchos casos a la población femenina, explica en algunos casos la disminución "histórica" de la fecundidad hasta los años sesenta-setenta, pero no justifica, en cambio, la aceleración de esta tendencia en la última década.

Con frecuencia el descenso de la fecundidad en Europa del Sur, se relaciona con el descenso paralelo de la nupcialidad (F.Munoz- Perez, 1987). En Aragón el descenso de la nupcialidad no es demasiado sensible respecto de épocas anteriores. Los indices de solteria son relativamente importantes, pero eso no es nuevo en la región. La nupcialidad sigue siendo la principal fórmula de creación de celulas familiares. Otras uniones de caracter estable no representan mas que el $0,48 \%$ de la población femenina, que es una 
tasa muy baja si se compara con el porcentaje de uniones estables no matrimoniales de otras regiones.

El porcentaje de mujeres casadas, la edad de contraer matrimonio (de 20 a 24 años el $58,16 \%$, y de 25 a 29 años en el $25,59 \%$ del total), y su duración media (de 10 a 14 años en el $42,70 \%$ de las mujeres casadas y de 15 a 19 en el $25,40 \%$ ), deberían ser causa de una mayor fecundidad.Como no es así, hay que concluir que la fecundidad real está muy lejos de la fecundidad natural, lo cual indica que ésta se halla fuertemente controlada por razones de mentalidad inherentes al cambio social, o por otro tipo de razones socioeconómicas, salvo que se demuestre que ha habido una disminución biológica de la fecundidad, como parecen apuntar las últimas encuestas sobre fecundidad.

En efecto, según la encuestas de fecundidad anteriormente citadas, Aragón tiene un porcentaje de esterilidad femenina (no voluntaria), relativamente importante en el contexto español. Desconocemos sus causas y hasta qué punto es algo propio de la población aragonesa. Aún así este hecho no es suficiente para explicar por completo la fecundidad actual. El riesgo de embarazo supera el $85 \%$ de las mujeres casadas y el $89 \%$ de todas las mujeres.Eso apoya la hipótesis de que las prácticas de control de la natalidad están muy generalizadas. La encuesta de fecundidad advierte que el conocimiento de los métodos anticonceptivos es general. Las campañas de difusión de estos métodos han sido muy eficaces y en la mayoría de los núcleos urbanos las autoridades municipales sostienen centros de "orientación familiar" que se traducen en centros para el control de la natalidad.

Así, las prácticas de control de natalidad están muy generalizadas. Solo un tercio de todas las mujeres aragonesas no había utilizado alguna medida de control, según la encuesta de 1985. Entre las casadas el control es mayor pues solamente el 11,04\% no habían utilizado ningún método. Dado que la natalidad aragonesa es sobretodo matrimonial, se deduce que hay un fuerte control sobre la misma dentro del matrimonio, lo cual pone de manifiesto, una vez más, la existencia de una actitud restrictiva respecto a la natalidad, como ya se ha dicho.

Las edades en las que se producen la mayor parte de los nacimientos, el número de hijos por mujer y la relación positiva entre mayor natalidad y menor instrucción, corroboran lo dicho. 


\section{Edad media de la matemidad en las capitales}

\begin{tabular}{lccc} 
& $\underline{1975}$ & $\underline{1981}$ & $\underline{1986}$ \\
Huesca & 28,15 & 27,93 & 9,90 \\
Zaragoza & 28,17 & 27,86 & 29,35 \\
Teruel & 27,80 & 27,50 & 29,25 \\
\hline
\end{tabular}

Fuente: I.N.E.

La edad media de la maternidad se halla entre los 27 y 28 años para la población de las diferentes comarcas aragonesas, mientras que se retrasa algo mas en las capitales. A diferencia de la tendencia española, en Aragón la natalidad se retrasa respecto del comienzo de la fecundidad. Ello es consecuencia, en primer lugar, del retraso de la edad de contraer matrimonio; y luego por el mayor control de la natalidad.

A partir de 30 años la reducción voluntaria de la natalidad es elevada. El $92 \%$ de las mujeres casadas declaraban en 1985 que no tenían intención de tener un hijo en el futuro. Tambien las mujeres mas jóvenes se cuestionaban esa posibilidad en mas del sesenta por cien de los casos.

A la vista de estos hechos hay que concluir que se da un comportamiento negativo y generalizado respecto de la fecundidad. La generalización del uso de los métodos anticonceptivos fue propiciada por el cambio sociopolítico sobrevenido en los años ochenta. Las jóvenes generaciones de procreadores vieron liberalizada la utilización de métodos anticonceptivos y pudieron utilizar las llamadas oficinas de planificación familiar. La única política desarrollada respecto a la población ha sido la de la limitación de la natalidad, como ya se ha indicado, sin caer en la cuenta de que esa política tendría consecuencias muy negativas en algunas regiones.

Desde el punto de vista demográfico, esto es claramente negativo para Aragón. La política de limitación de nacimientos ha prosperado en una población numéricamente disminuida y estructuralmente envejecida, que ve así muy comprometido su futuro. 
TASAS PROV. DE FECUNDIDAD POR EDAD $(, 000)$
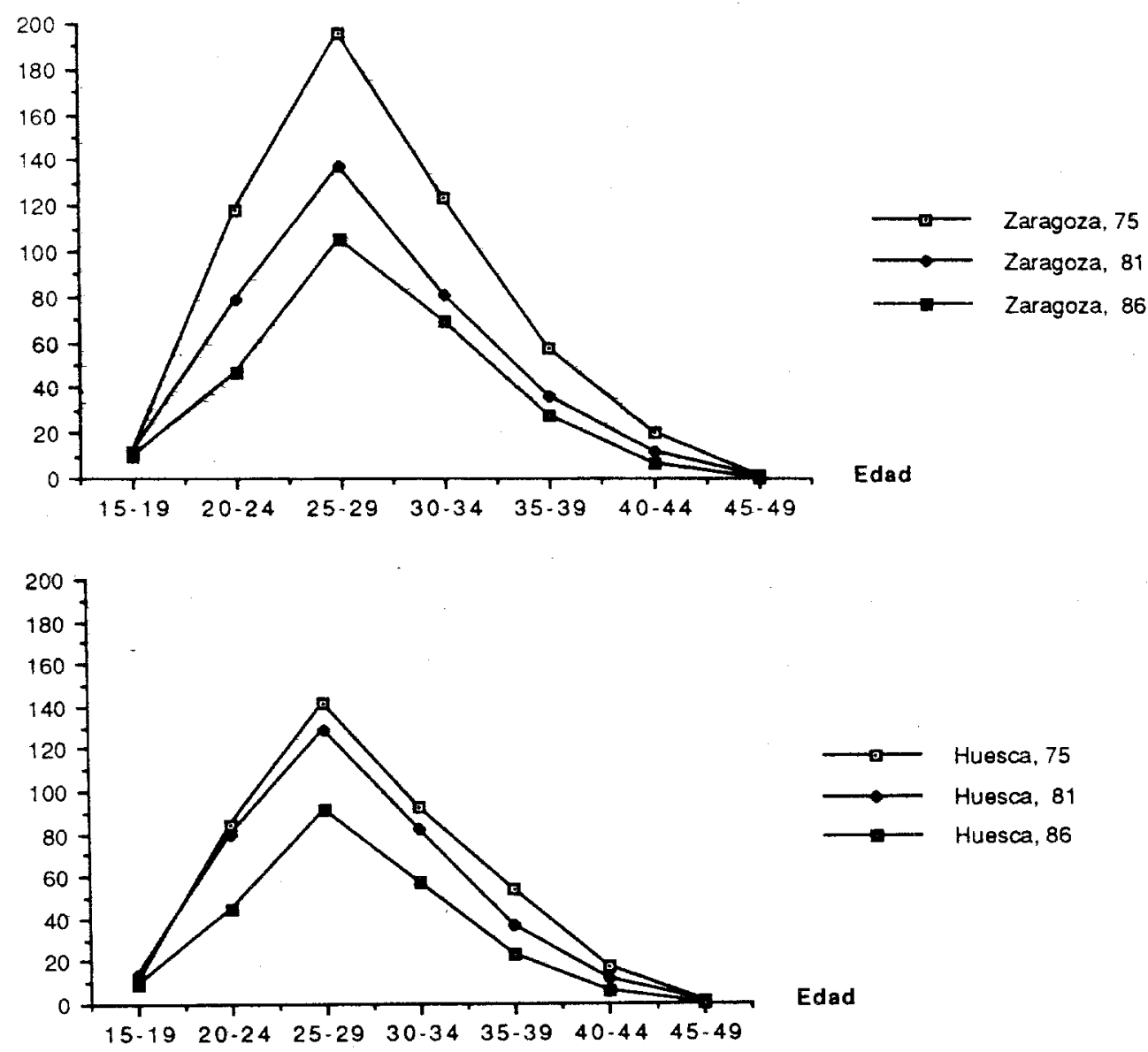

Edad
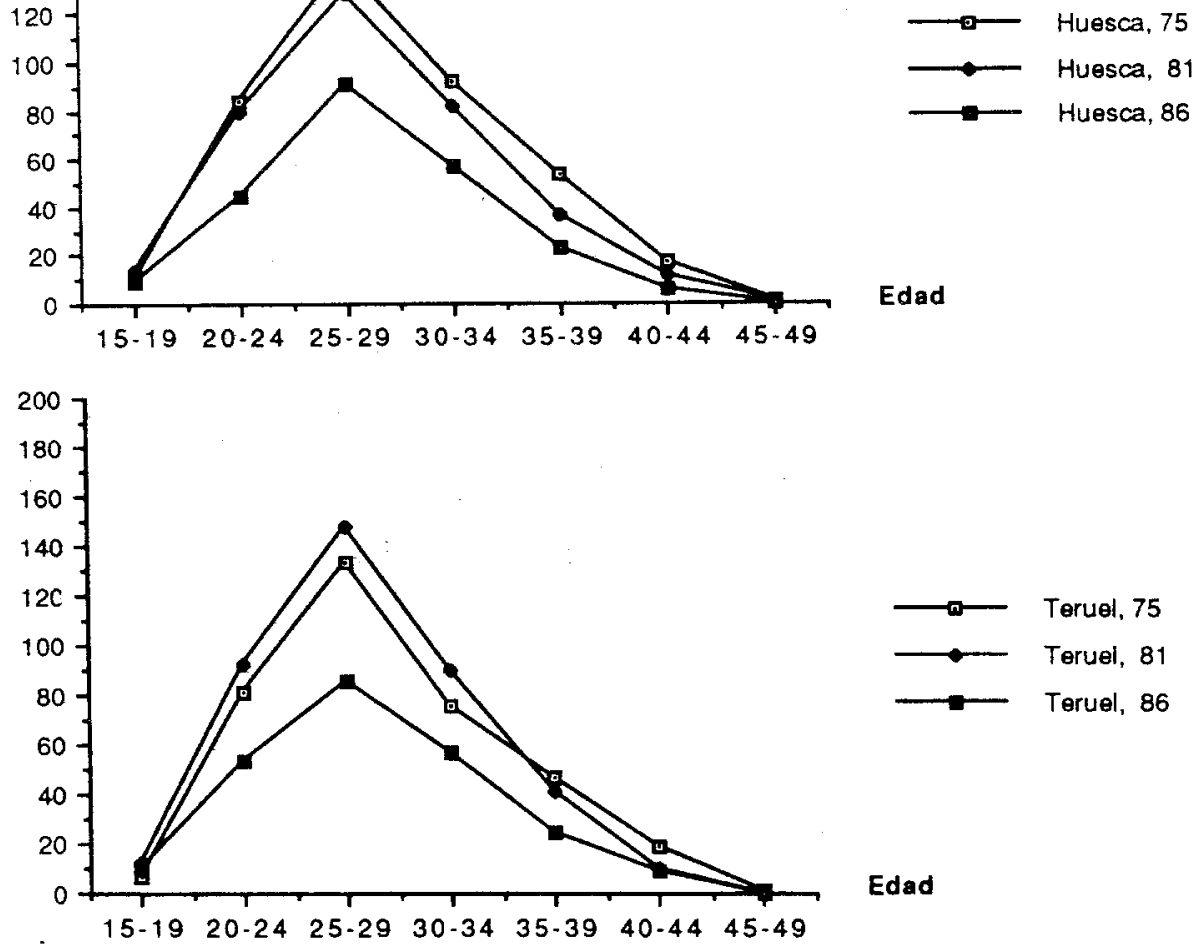

$\longrightarrow$ Teruel, 75

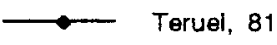

Teruel, 86 
Bien es verdad que en última instancia es el comportamiento individual el que decide la adopción o no de tales medidas y ello dentro de la valoración que se haga de la familia y de los hijos; del concepto de las relaciones hombre-mujer, y del nivel cultural. En definitiva la mentalidad de los posibles padres (D.Noin, 1988). La mentalidad aragonesa ha evolucionado en este sentido, de manera que el comportamineto demográfico no cambiará hasta que no llegue una nueva generación de progenitores con mentalidad diferente. Ello no se producirá probablemnte hasta el año 2000 , pero para entonces ya será seguramente tarde.

\section{ALGUNAS CONCLUSIONES}

Varias son las conclusiones que se derivan de este análisis. A modo de resumen señalaremos algunas de ellas:

- Aragón es una de las regiones españolas con menor fecundidad junto con el País Vasco, Asturias y Galicia.

- Dentro del conocido retraso en la caida de la fecundidad que caracteriza a la Europa meridional respecto de la Europa occidental, en Aragón el descenso de la fecundidad debido al envejecimiento de la población derivado de la emigracion, se produjo muy pronto.

- Actualmente se ha entrado en un proceso de fecundidad dirigida. Factores de comportamiento favorecidos por el cambio político y socioeconómico, se suman a los factores estructurales.

- Disminuye el tamaño de las familias siguiendo las características de la población europea. Pocas familias numerosas, aumento de las parejas sin hijos (mas del $30 \%$ ), y dominio de hogares con uno o dos hijos.

- Parece que la tendencia se va a mantener dado el alto porcentaje de parejas que se cuestionan incluso la posibilidad del primer hijo, tal vez por los problemas que la maternidad originaría en caso de divorcio (autorizado desde 1978). 
- El enome descenso de la fecundidad compromete el futuro demográfico. Podría decirse que Aragón ya está en lo que Airk Van de Kaa denomina la Segunda Transición Demográfica, en la medida en que se prevé un decrecimiento de su población.

\section{BIBLIOGRAFIA:}

-COALE,A.J.(1969): "The decline of Fertility in Europe from the French Revolution to World War II". In "Fertility and Family Planning". Ed. by S.J. Berhman, L. Corsa and R. Freedman. Ann Arbor, Michigan: University of Michigan Press.

-COWARD,J.(1986): "Fertility Patterns in the Modern World" in Population Geography.Progress and Prospect. Pp. 58-94. Ed.M.Pacione.Croom Helm. London.

-EASTERLIN,R.A.(1975): "An Economic Framework for Fertility Analysis". En "Studies in Family Planning" 6(3). Pp. 54-63.

-EASTERLINE, R.A. et alt.(1978): "Demographic Influences on Economic Stability: the U.S. experience". Population and Development Review 1. Pp.1-22.

-GARCIA,A.-CRESPO,M.J.(1988): "Chomage et Fécondité Post- Transitionelle en Espagne". Colloque sur La Transition Démographique dans les Pays mediterraneens. Université de Nice.

-GRAUMAN,J.(1960): "Comment" in "Demographic and Economic Change in Developed Countries". Princeton. Pp. 275-284.

-HIGUERAS,A.-FAUS,M.C.(1988):"Estructura y Perspectivas Demográficas de Aragón". T.II. Ed. Ibercaja.

-I.N.E.:- "Movimiento Natural de la Población Española".Varios años.

- "Boletines Mensuales de Estadística".Varios años.

- "Anuario Estadístico de España".Varios años.

-MUNOZ-PEREZ,F.(1987): "Le déclin de la Fécondité dans le Sud de L'Europe". Population 6. Pp.911942. INED.

-NOIN,D.(1988): "La Transition de la Fécondité en Europe du Sud". Colloque sur La Transition Démographique dans les Pays Mediterranéens. Université de Nice.

-PEREZ MOREDA, V.(1984): "Evolución de la Población Española desde finales del antiguo regimen".Papeles de Economía española. Ed.Fundación Fondo para la Investigación Económica y Social. Confederación Española de Cajas de Ahormo. Pp 20-38.

-SANZ, A.-TERAN, M.(1988): "Las disparidades sociales regionales" en Papeles de Economía Española. Ed. Fundación Fondo para la Investigación Económica y Social. Confederación Española de Cajas de Ahorro. Pp 82-114.

-TAPINOS, G.(1985): "Éléments de Démographie". Armand Colin. Paris. 
-WOODS,R.(1979): "Population Analysis in Geography". Longman. London .

-WUNSCH, G.(1987): "Tendances de la Fécondité en Europe". De la Description a L Explication". II Congreso Mundial Vasco. Vitoria. Pp.82-98.

INDICE SINTETICO DE FECUNDIDAD (N" de hijos por mujer). 1981

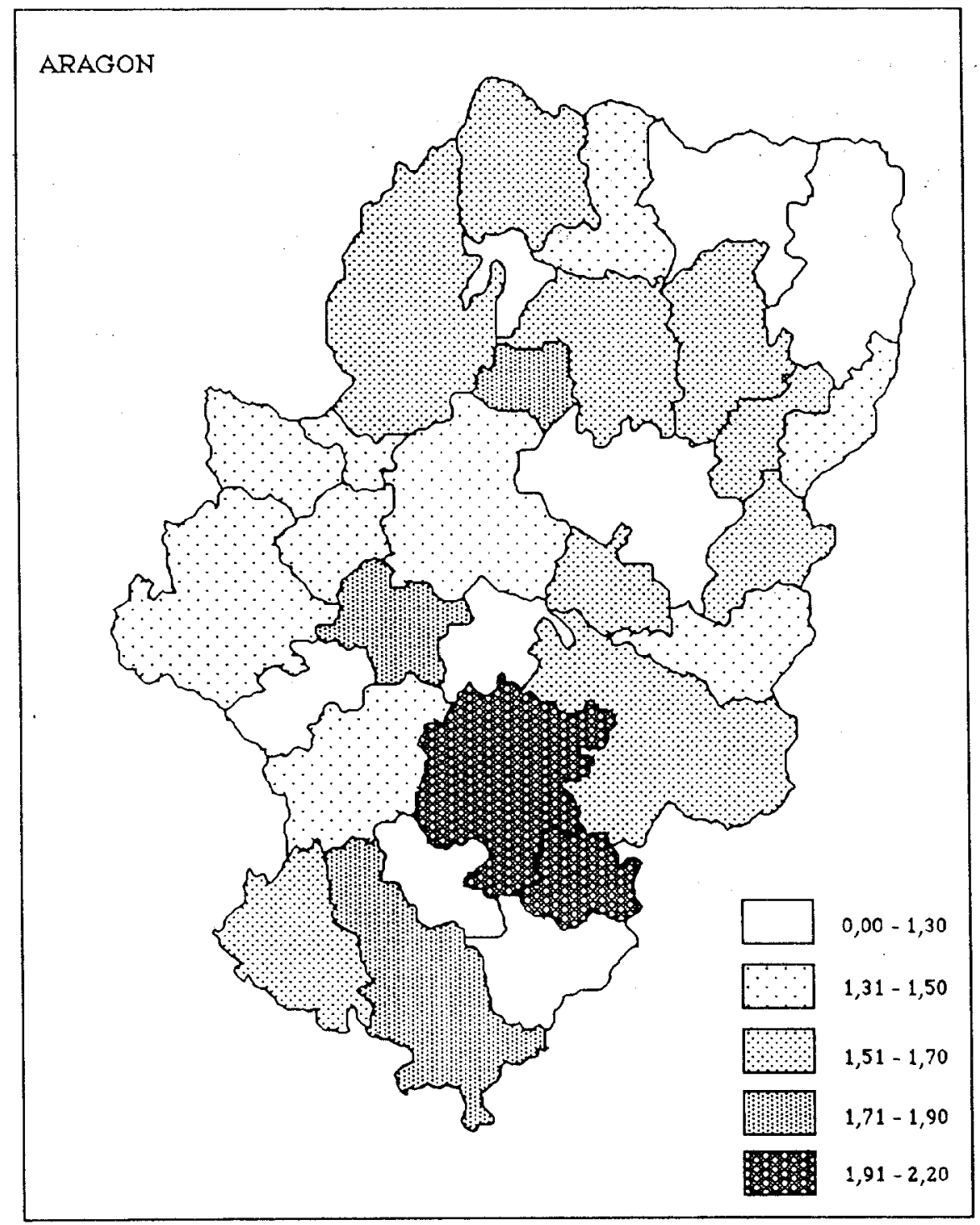




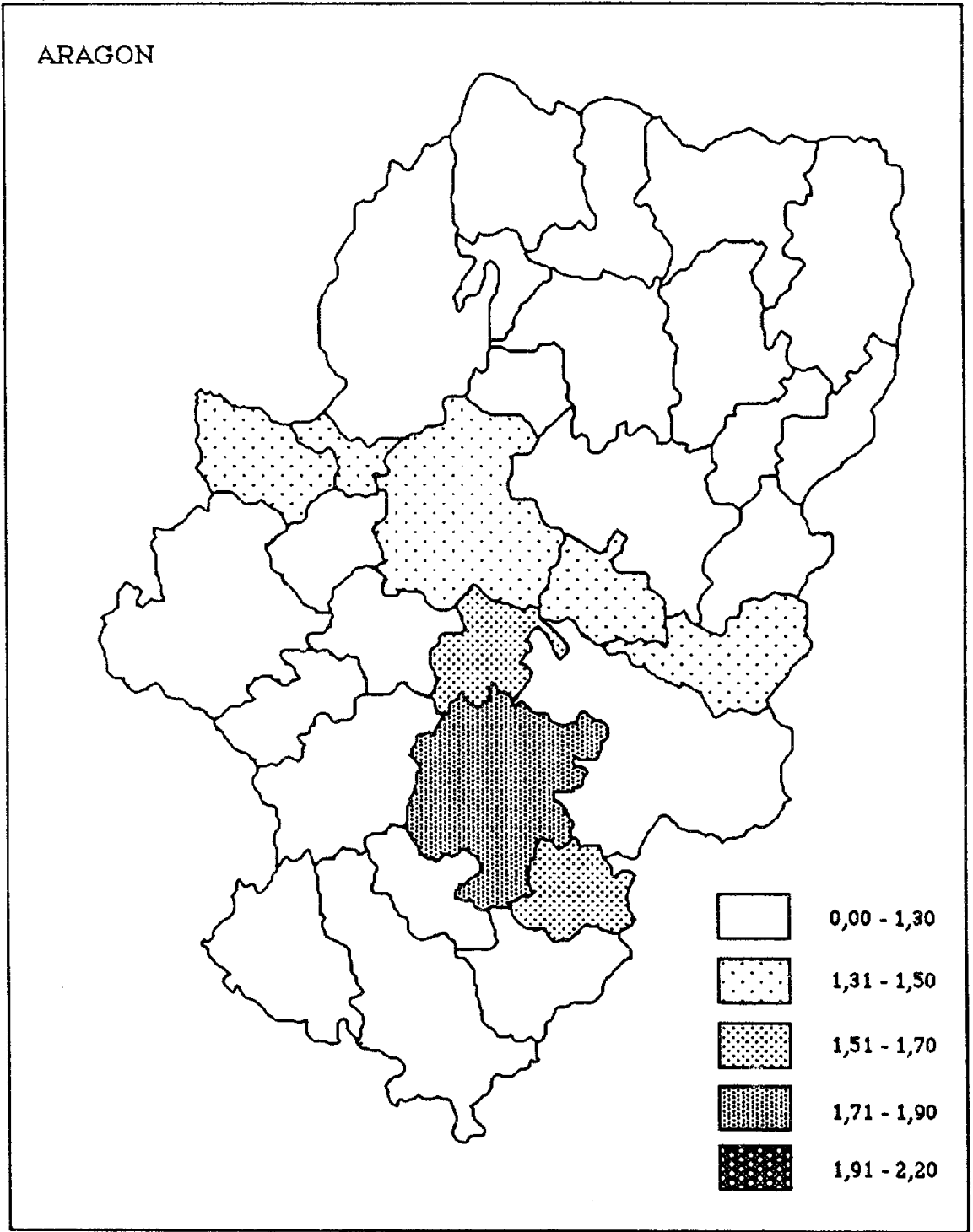

